EL EFECTO DE LA POLÍTICA MONETARIA

SOBRE EL COMPORTAMIENTO DE LOS BANCOS COMERCIALES:

EL MERCADO “LECHERO”. EN MEXICO

Lorenza Martínez Trigueros ${ }^{1}$

DOCUMENTO DE INVESTIGACIÓN No. 9506

AGOSTO, 1995

\footnotetext{
${ }^{1}$ La autora es candidato doctoral del Massachussets Institute of Technology. Este trabajo fue elaborado durante su estancia como investigadora invitada del Banco de México en el verano de 1995. Las opiniones contenidas en el documento representan los puntos de vista de la autora y no necesariamente reflejan los de las instituciones arriba referidas.
} 


\title{
EL EFECTO DE LA POLÍTICA MONETARIA \\ SOBRE EL COMPORTAMIENTO DE LOS BANCOS COMERCIALES: EL MERCADO "LECHERO". EN MEXICO
}

\author{
LORENZA MARTINEZ TRIGUEROS \\ AGOSTO, 1995
}

DOCUMENTO DE INVESTIGACIÓN No. 9506

\section{RESUMEN}

Con base en los cambios recientes en la regla de intervención del Banco de México, el presente documento analiza el efecto de implementar un mercado interbancario de créditos para cuadrar los balances resultantes en la cuenta del sistema de pagos, después del cierre del día. Se muestra que, abrir un mercado de créditos después de conocer los choques exógenos sobre la demanda de dinero diaria conduce a un Pareto superior. Mas aún, el segundo mejor se alcanza cuando la regla de intervención del Banco Central consiste en ofrecer créditos o depósitos para saldar la cuenta del sistema bancario completo. Se muestra que, imponer una tasa punitiva para sobregiros a los bancos comerciales mayor que la tasa activa, es suficiente para evitar que los bancos se sobregiren voluntariamente. Una conclusión importante de este artículo es que los frecuentes sobregiros registrados durante 1994 no fueron debidos a un problema del mercado "lechero", sino que podrían estar más cercanamente relacionados con las características de la política cambiaria vigente.

\section{INTRODUCCIÓN}

En marzo de 1993, el Banco de México propuso la apertura de un mercado interbancario vespertino de crédito, el cual operaría después de que el mercado convencional cerrara. El objetivo de este mercado consistía en ampliar el tiempo de intercambio tanto a los bancos comerciales como al banco central, una vez que los choques en la demanda de dinero de base fuesen asimilados. La implementación de dicho mercado fue un logro importante, ya 
que el error de estimación de la demanda diaria de dinero de base por parte del banco central era generalmente muy grande.

Así mismo, el mercado fue creado para mejorar el sistema financiero en dos aspectos. El primero se logró permitiendo al banco central corregir cualquier error en su intervención durante el día, ocasionada por algún choque no anticipado. El segundo tiene que ver con la incertidumbre que experimentan los bancos comerciales en cuanto al balance resultante de las operaciones interbancarias diarias. Tal balance es asimilado por los bancos hasta muy tarde o incluso el día siguiente, por lo que podían incurrir en un sobregiro con el banco central sin saberlo. En este sentido, es importante resaltar que este nuevo mercado contribuyó a resolver un problema operativo. Esto es, no existía un mecanismo capaz de registrar más rápidamente el balance interbancario. Por esta razón. Entre otras, la importancia de este mercado está supeditada a la innovación tecnológica, Adicionalmente, es importante subrayar que existe una fuente de incertidumbre difícil de eliminar, la demanda de billetes y monedas. Pese a ello, a medida que el error se reduce, la importancia del mercado "lechero" también se reduce. Se han dado algunas mejoras en las estimaciones desde 1993, pero el error de estimación del dinero de base aún es muy grande.

Este mercado interbancario, llamado "lechero", opera créditos interbancarios nocturnos con valor al día anterior. Esto es, cada mañana de las 8:00 a.m. a las 9:30 a.m. un mercado de crédito interbancario opera para saldar las cuentas de los bancos comerciales en el Banco de México correspondientes al día anterior. Dada la regla de intervención explicada previamente, se corrigen dos desequilibrios consecuencia de la incertidumbre en la demanda diaria de dinero de base. Primero, el desequilibrio de todo el sistema por el "error" del banco central en la predicción de la demanda de dinero de base. Segundo, el desequilibrio experimentado en la cuenta de un banco dado y que tiene su contraparte en la cuenta de otro banco.

Desde marzo de 1993 y hasta marzo de 1995, el Banco de México solía intervenir en este mercado, saldando el balance de todo el sistema. Esta política se estableció para reducir la volatilidad de tasas de interés. Por un lado, cualquier choque agregado no anticipado en la 
demanda de dinero podía ser acomodado por el banco central sin afectar las tasas de interés. Por otro, cuando choques idiosincráticos colocaban a algún banco en posición estratégica, el conocimiento de una futura intervención reducía el poder de negociación de ese banco y facilitaba las transacciones diarias. Sin embargo, esta regla de intervención, como cualquier otra, altera los incentivos de los bancos comerciales de mantener reservas en el banco central.

Como habrá de mostrarse a través de un modelo en la sección 3, las mejoras obtenidas con el mercado "lechero" no se relacionan con la propuesta estabilización de las tasas de interés. En nuestro análisis, obtenemos el resultado común de la literatura financiera que la volatilidad de tasas está lejos de ser perversa.

La virtud de esta regla de intervención en el mercado "lechero" radica en que el banco central absorbió todo el riesgo derivado de la falta de información sobre la demanda diaria de dinero de base, asegurando a los bancos comerciales tanto de choques agregados no anticipados en dicha demanda, como de contracciones voluntarias en el dinero de base. Dada esta política de intervención, no existía razón para que los bancos mantuvieran reservas en exceso en el banco central, aumentando el bienestar social. Friedman y Schwartz (1963) destacan la importancia de tener dinero productivo y el efecto negativo de mantener reserves en exceso ya sea en los bancos comerciales o como depósitos en el banco central.

Después de marzo de 1994, el sistema bancario comenzó a incurrir en sobregiros frecuentemente. El Banco de México interpretó tal situación como un problema del mercado "lechero", como si éste hubiese incentivado el sobregiro de los bancos. La presunción del Banco de México de estar acomodando más crédito y menos demanda de dinero, obligó a dicha institución a cambiar la regla de intervención en marzo de 1995. Desde entonces, la tasa punitiva aumentó y las intervenciones de la institución se limitaron a dos ocasiones aisladas. 
El propósito de este artículo es definir una política monetaria óptima cuando la demanda diaria de dinero de base experimenta incertidumbre. Para ello se construye una función de bienestar social y así obtener el nivel óptimo de reservas bancarias. El artículo procede a incorporar la asimetría de información entre los bancos comerciales y el banco central para determinar la política óptima que permita alcanzar el segundo mejor. Los bancos privados deciden un nivel de crédito y un nivel de reservas planeado. Dado que el banco central no observa los créditos en forma separada de los depósitos, sino únicamente los sobregiros en la cuenta, la regla de intervención tiene efectos perversos sobre los incentivos de los bancos privados.

El modelo incorpora el efecto que distintas reglas de política económica tienen sobre las decisiones de crédito de los bancos comerciales. Por otra parte, también se obtiene la política óptima para el banco central, la cual resulta ser superior a la política de no intervención. Del modelo se obtiene que la regla de política adoptada de marzo de 1993 a marzo de 1995 era eficiente, pero no compatible con una política de tipo de cambio fijo. Ahora, cabe preguntarnos cuál regla de política es compatible con un régimen de tipo de cambio fijo no creíble. Más aún, es bien sabido que un sistema bancario nacional y un tipo de cambio fijo no son compatibles.

El artículo está organizo como sigue. La sección 2 narra las recientes reformas en torno a la regla de intervención del banco central, así como la evidencia empírica referente a la política monetaria en México y sus efectos. La tercera sección presenta un modelo simple para abordar dos preguntas. Primero, cómo afectan las diferentes reglas de intervención del banco central a las estrategias adoptadas por parte de los bancos comerciales y, segundo, cuál es la regla de intervención óptima para el banco central.

Una conclusión importante del modelo es que resultaría óptimo para el banco central el proveer fondos adicionales o tomar crédito adicional en el mercado de dinero diario sólo si los bancos privados experimentan incertidumbre en su oferta de depósitos. De otra manera, el resultado de mercado coincide con el óptimo y el banco central no tiene incentivos a intervenir. No resulta difícil creer que la situación más realista es aquella en la que los 
bancos comerciales experimentan incertidumbre y, por tanto, la regla de intervención adoptada por el Banco de México era óptima.

\section{POLÍTICA MONETARIA Y EL MERCADO "LECHERO” EN MEXICO}

En los últimos años, al menos desde 1989, la política monetaria adoptada por el Banco de México se ha enfocado a proporcionar la liquidez necesaria para el sistema. Esto es, el banco central estima diariamente una demanda de dinero de base y la inyecta (o retira) al sistema a través de operaciones de mercado abierto. Al mismo tiempo, el banco central ha intentado dar una mayor transparencia a sus operaciones.

En 1989, el Banco de México eliminó el encaje legal e implementó un coeficiente de liquidez. Este coeficiente establecía que los bancos comerciales debían mantener en el banco central un monto mínimo de bonos gubernamentales proporcional a sus obligaciones. El objetivo de esta reforma era reducir los costos financieros excesivos de los bancos que fuesen considerados innecesarios.

No es sino hasta septiembre de 1991 que se elimina el coeficiente de liquidez sobre obligaciones en pesos y se reduce la tasa de interés para sobregiros pequeños e infrecuentes. La explicación que daba el Banco de México considera el hecho de que la oferta de bonos gubernamentales iba disminuyendo, a la vez que la demanda por depósitos aumentaba. Lo anterior requería de un mayor monto de bonos e inducía una reducción ficticia en las tasas de interés. Más aún, durante agosto de 1991, algunos bancos comerciales utilizaban sus fondos de reserva sobre el coeficiente de liquidez para cubrir la demanda por créditos. Al final del mes, los bancos estaban tan deseosos de reestablecer sus reservas que la tasa de interés sobre depósitos experimentó un fuerte incremento, mientras que la tasa de bonos gubernamentales cayó. Así, el banco central decidió que era el momento apropiado para eliminar el coeficiente de liquidez.

Tales cambios en los requerimientos sobre reservas, junto con la liberación de las tasas de interés pagadas a las cuentas de cheques, cuentan para el marcado incremento en M1 del 
último cuatrimestre de 1991 al segundo cuatrimestre de 1992. Durante 1991, la tasa de crecimiento para M1 fue de $119.7 \%$ y de $17 \%$ durante 1992 . De cualquier manera, este severo incremento no tuvo efectos significativos en la tasa de inflación puesto que representó mayormente transferencias de cuentas de ahorro o depósitos a cuentas de cheques.

La creencia por parte del Banco de México en que es mejor instrumentar la política monetaria mediante operaciones de mercado abierto que mediante el encaje legal o el coeficiente de liquidez también apoya los cambios institucionales ya mencionados. En oposición a esta línea, en junio de 1991 el banco central estableció un coeficiente de liquidez sobre depósitos en moneda extranjera. Después de agosto, tal requerimiento se tornó aún más restrictivo. El banco central intentaba frenar el crecimiento acelerado de obligaciones en moneda extranjera en bancos comerciales mexicanos.

Durante 1991, la política monetaria se orientó a la esterilización de grandes entradas de capitales a través de reducciones en el crédito doméstico. La esterilización fue sólo parcial, y el dinero de base se incrementó. El banco central prestó 5,300 billones de Nuevos Pesos mediante subastas de crédito de corto plazo en ese año. 


\section{Gráfica 1}

Saldo en Cuenta Única antes del Mercado Matutino

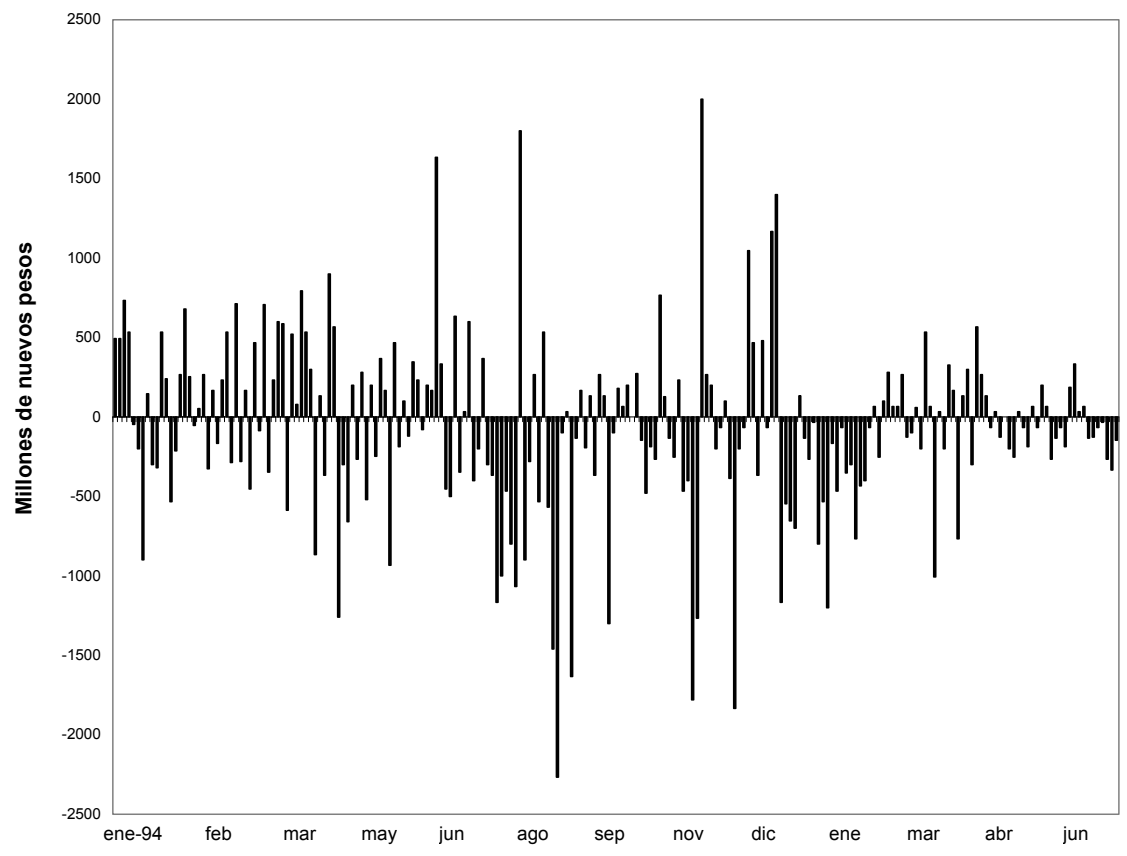

\section{Gráfica 2}

Saldo en Cuenta Única después del Mercado Matutino

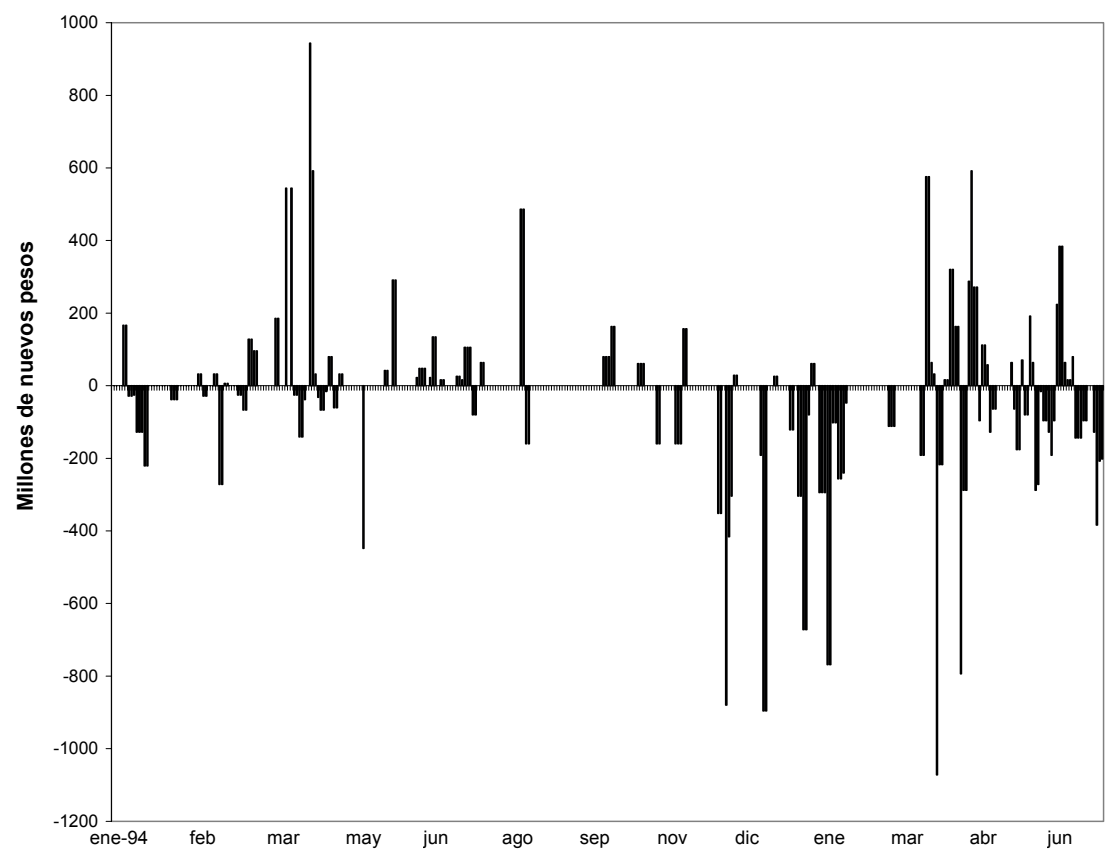




\section{Gráfica 3}

Tasa Interbancaria y Tasa de Descuento

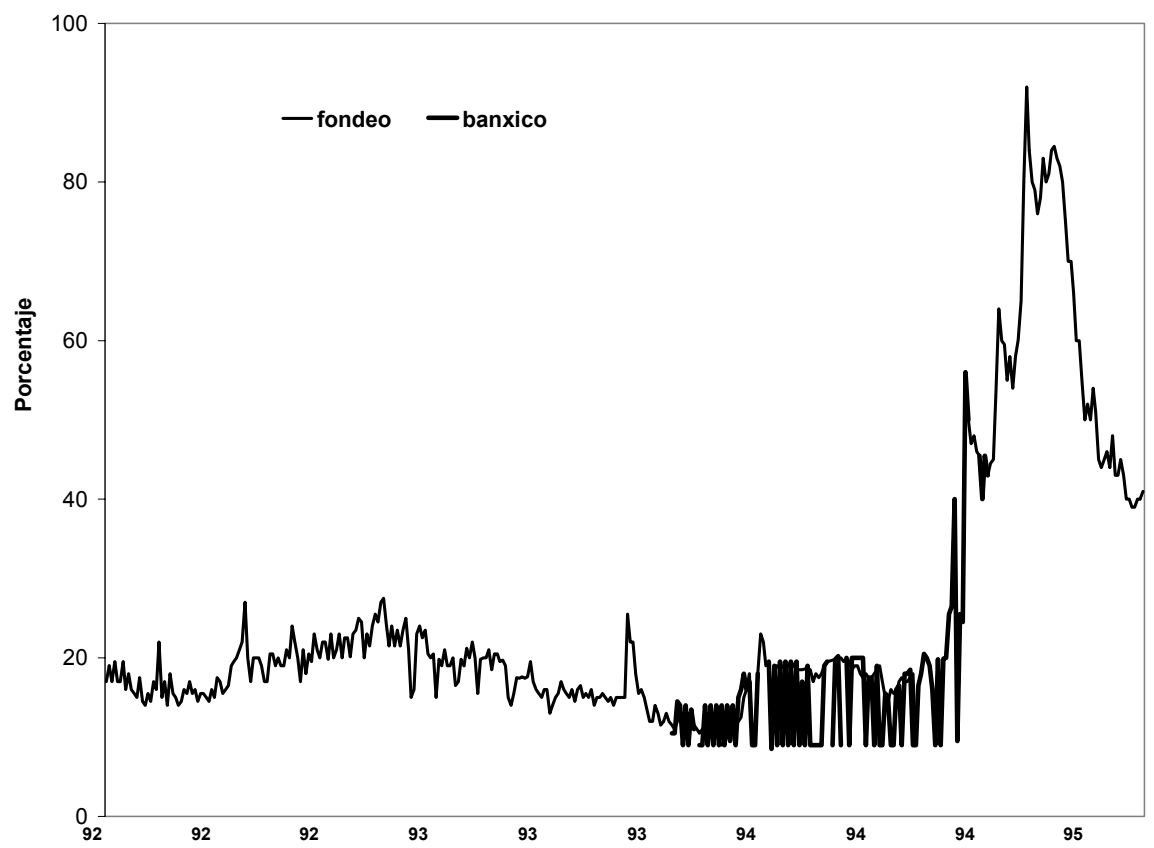

Es hasta marzo de 1993 cuando el mercado "lechero" da inicio y cuando el banco central empieza a intervenir en el mercado matutino de crédito con valor al día anterior. Por ello, los bancos comerciales redujeron sus depósitos en el banco central a niveles tales que el dinero de base terminó casi al nivel de billetes y monedas. Con todos estos cambios, el banco central buscó establecer el marco apropiado para la consecución de sus objetivos sólo mediante operaciones de mercado abierto.

El banco central decidió abrir el mercado interbancario matutino de créditos para reducir la volatilidad de tasas de interés y amplificar su control sobre las variables monetarias. Bajo este nuevo sistema, el banco central tenía la posibilidad de intervenir en tal mercado para eliminar cualquier desequilibrio no deseado en las cuentas de los bancos comerciales en el banco central. Aún cuando no se estableció explícitamente, el Banco de México terminaba saldando el mercado todos los días, lo cual puede apreciarse si comparamos los balances en las gráficas 1 y 2 . Por lo que respecta a la tasa de descuento del banco central, esto es, la 
tasa a la que el Banco de México presta dinero, en la figura 3 podemos observar que ésta se encontraba, en la mayoría de las veces, por arriba de la tasa interbancaria.

Los depósitos de los bancos comerciales en el banco central cayeron de \$1,957 millones de Nuevos Pesos en diciembre de 1992 a cerca de cero en diciembre de 1993. Paralelamente a esta contracción, el banco central redujo el crédito doméstico para evitar posibles efectos monetarios derivados de tal cambio institucional.

Como se explicó anteriormente, en marzo de 1995 el banco central cambió su política e implementó un nuevo sistema. A partir de entonces, el banco central ha intervenido en el mercado "lechero" sólo dos veces debido a que hubo una enorme demanda no anticipada de billetes y monedas. Ahora, los balances bancarios son acumulados durante un mes y deben mantener un depósito promedio no negativo.

\section{EL MODELO}

El modelo supone dos tipos de agentes: el banco central (BC) y el sistema bancario, integrado este último por bancos comerciales. Los bancos comerciales enfrentan una demanda por crédito y una oferta de depósitos, ambos función de la tasa correspondiente ofrecida por el banco y por aquellas ofrecidas por el resto de los bancos. Se supone que los bancos deciden los momentos de crédito y reservas en función de la oferta de depósitos y la incertidumbre de la misma. Cabe hacer notar que el nivel de depósitos no es una variable de control para los bancos, sino que éstos fijan sólo las tasas de interés mientras que la cantidad depende únicamente de la realización del choque. Si los bancos pudieran determinar el nivel de depósitos, observaríamos cambios en las tasas, pero nunca sobregiros. Es bien sabido que sobregirarse en el sistema bancario puede ser una situación usual, aunque algunas veces adquiere dimensiones que resultaría difícil creer que fueron deliberadas. Como explicaremos a continuación, la necesidad de un prestamista de última instancia en las operaciones diarias se deriva en forma directa de la presencia de incertidumbre en los depósitos. 
Los bancos comerciales, considerando la regla de intervención del banco central, eligen la proporción de activos mantenida en forma de reservas que maximice ganancias. La demanda por crédito para el banco $i$ está dada por:

$$
C_{i}=\frac{1}{n}\left[a_{c}+c_{o} r_{i}^{c}+c_{1} \sum_{j=1}^{n}\left(r_{i}^{c}-r_{j}^{c}\right)\right]
$$

donde $n$ es el número de bancos en el sector $r_{t}^{c}$ es la tasa de interés sobre créditos por el banco i.

La oferta de depósitos está dada por:

$$
D_{i}=D_{i}+\epsilon_{i}
$$

donde $D_{i}=\frac{1}{n}\left[a_{d}+d_{o} r_{i}^{d}+d_{1} \sum_{j=1}^{n}\left(r_{i}^{d}-r_{j}^{d}\right)\right] \mathrm{y} \in$, es un choque aleatorio con valor esperado igual a cero. Para efectos de simplificación, se asume que, $\in$, sólo puede tomar dos valores: $-\in$ con probabilidad $p, \mathrm{y} \in$ con probabilidad 1-p. La realización del choque es asimilada luego de haber escogido las tasas activa y pasiva. Las reservas bancarias se dividen en planeadas y no planeadas. Las reservas planeadas están dadas por la diferencia entre depósitos y créditos, suponiendo que la realización del choque sea cero:

$$
X_{i} \equiv D_{i}-C_{i}
$$

Las reservas no planeadas están dadas por la suma de las reservas planeadas más la realización del choque:

$$
X_{i} \equiv X_{i}+\epsilon_{i}
$$




\subsection{LA FUNCION DE BIENESTAR SOCIAL}

Antes de analizar el problema de los bancos, se determinará la solución óptima desde una perspectiva social. Este paso es importante para encontrar racionalidad a las intervenciones del banco central cuando operan los bancos comerciales. En la literatura sobre regulación financiera es común el uso de formas reducidas para la función objetivo de la autoridad financiera. En general, éstas terminan siendo funciones ad-hoc para cierto propósitos, y difíciles de derivar como función objetivo para una autoridad racional. Para evitar la falta de claridad que podría venir de incluir directamente la varianza de tasas de interés, las ganancias del sistema bancario o la varianza de la cantidad de dinero en el ejercicio de optimización, se hará una derivación completa de la función objetivo.

La función objetivo del banco central será el excedente del consumidor en el mercado de créditos. Es decir, con la demanda por créditos y la oferta de depósitos, la función de bienestar será el área entre estas dos curvas.

\section{Gráfica 4}

El Mercado de Crédito

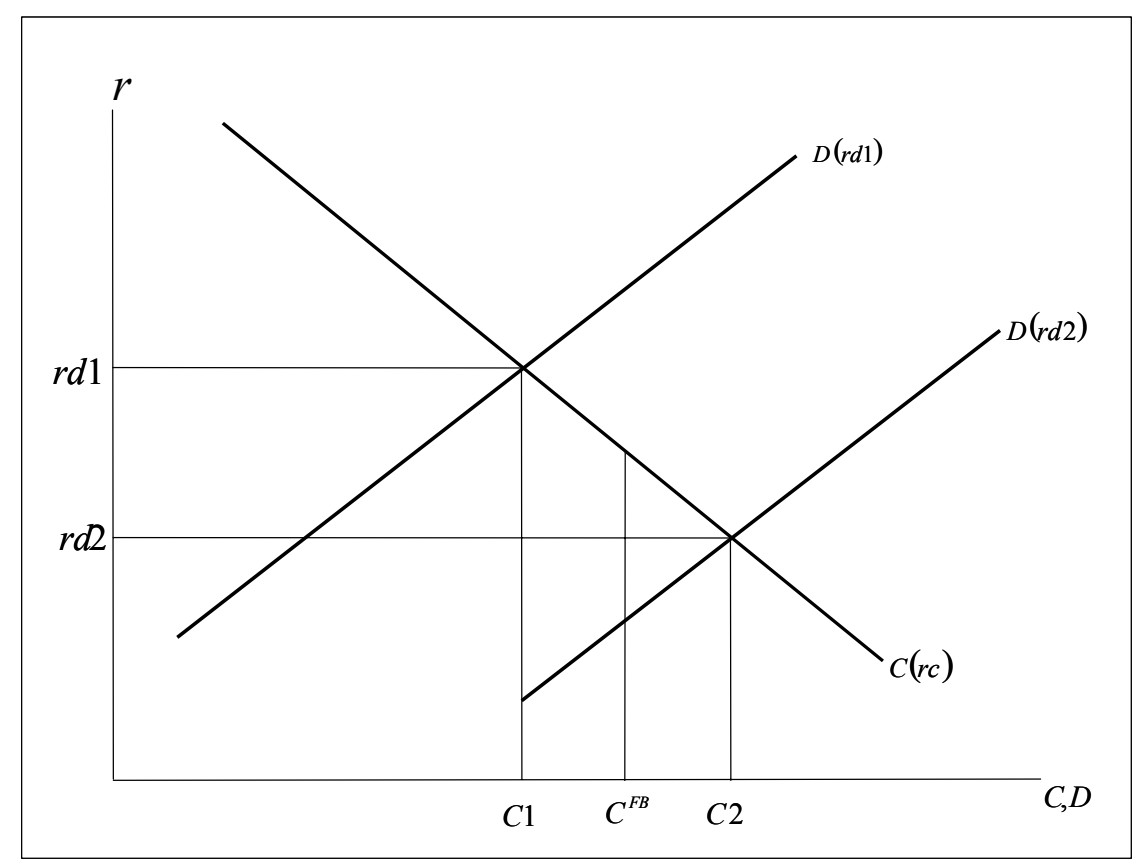


Considerando el caso en que las decisiones de crédito son tomadas antes de la realización del choque, la expectativa de bienestar social está dada por:

$$
E(S W)=p S W_{1}+(1-p) S W_{2}
$$

donde:

$$
\begin{aligned}
& S W_{1}=\int_{0}^{c^{f h}}\left[r^{c}(C)-r^{d 1}(C)\right] d C \\
& S W_{2}=\int_{0}^{c^{f b}}\left[r^{c}(C)-r^{d 2}(C)\right] d C-\int_{C^{F B}}^{C_{2}}\left[r^{c}(C)-r^{d 2}(C)\right] d C
\end{aligned}
$$

c es el monto de crédito, $r^{d}$ y $r^{d 2}$ son el inverso de la oferta de depósitos, cuando ha experimentado un choque negativo y positivo respectivamente.

$$
r^{d 1}=\left(D-a_{d}+\in\right) / d_{0} \quad r^{d 1}=\left(D-a_{d}+\in\right) / d_{0}
$$

donde $r^{c}$ es el inverso de la demanda de crédito y el $c 1$ es el monto de crédito al cual la oferta de depósitos es igual a la demanda por crédito, cuando la última experimentó un choque positivo.

Maximizando el bienestar esperado con respecto a $c$, el nivel óptimo de crédito es:

$$
c^{F B}=\frac{a_{c} d_{0}+a_{d} C_{0}+C_{0} \in / 3}{c_{0}+d_{0}}
$$

Este nivel de crédito es mayor que aquel que equilibra la oferta de depósitos y demanda de crédito esperadas. Para adoptar tal nivel de crédito, el banco central tendría que subsidiarlo. Dado que éste es sólo un análisis de equilibrio parcial, no es suficiente para afirmar que el subsidio es eficiente. Por lo anterior, resulta más apropiado maximizar el bienestar esperado sujeto a una restricción presupuestal, donde el costo esperado de la regla de intervención sería cero. Entonces, el nivel de crédito óptimo restringido será: 


$$
c^{*}=\frac{a_{v} d_{0}+a_{d} c_{0}}{c_{0}+d_{0}}
$$

Por tanto, la intervención del banco central tendría lugar sólo si el equilibrio de mercado es diferente de $c^{*}$ y cuando la distorsión es el efecto de un problema de liquidez. Esta última condición es importante, si la distorsión es ocasionada por alguna otra razón como la falta de competencia, entonces el banco central no es la autoridad que debiera enfrentar el problema.

\subsection{SISTEMA BANCARIO.}

Para analizar las implicaciones de la intervención del banco central, derivemos el equilibrio de mercado bajo diferentes esquemas de información. Considérese un sistema con $n$ bancos, donde hay competencia de tasas de interés y los bancos son sustitutos imperfectos para el público.

Primero, se considera una situación en la que la oferta de depósitos no experimenta perturbación alguna. En esta situación, las tasas óptimas para los bancos son tales que no se mantienen reservas. Cualquier desviación a partir de $c^{*}$ se debe a la tasa de competencia, pero es fácil mostrar que, a medida que $n$ crece, el crédito agregado converge al nivel óptimo. El problema para el banco $i$ está dado por:

$$
\max _{C_{i}} E(n)=\left[r_{i}^{c}\left(C_{i}\right)-r_{i}^{d}\left(C_{i}\right)\right] C_{i}
$$

para simplificar, a partir de ahora se supondrá que: $a_{c}=c_{0}=c=d_{o}=d=1 \mathrm{y} a_{d}=0$.

$$
C_{i}=\frac{1}{2(1+n)}, \quad r^{d}=\frac{n}{2(1+n)}, \quad \mathrm{y} \quad r^{c}=\frac{1-n}{2(1+n)}
$$


La libre concurrencia garantiza que el número de bancos será lo suficientemente grande para alcanzar el nivel óptimo de crédito. Por lo tanto, es un entorno sin distorsiones, el banco central no tiene por que intervenir. Desde una perspectiva social, los bancos se anticiparían y tendrían un comportamiento óptimo. Bajo este escenario, nunca habría sobregiros. Sin embargo, tal no parece ser un caso realista. Más aún, si la oferta de depósitos experimenta distorsiones perfectamente anticipadas por los bancos comerciales, el bienestar social seria aún mayor que en el caso inicial.

Un caso más realista e interesante es aquel donde los bancos privados no puede anticipar choques de demanda por crédito. En ausencia de un prestamista de última instancia, y para altos costos de incurrir en una demanda de dinero de base que no pueda ser cubierta, los bancos mantendrían reservas suficientes.

$$
X_{i}=\epsilon, \quad \text { o } \quad D_{i}=C_{i}+\epsilon
$$

y el problema del banco sería ahora:

$$
\max _{C_{i}} E(n)=r_{i}^{c}\left(C_{i}\right) C_{i}-p\left[r_{i}^{d}\left(C_{i}+\in\right)\left(C_{i}\right)\right]-(1-p)\left[r_{i}^{d}\left(C_{i}+\in\right)\left(C_{i}+2 \in\right)\right]
$$

cuya solución simétrica estaría dada por:

$$
C_{i}=\frac{n-\in\left(1+n^{2}\right)}{2 n(1+n)}, \quad r^{d}=\frac{n+\in n^{2}+2 \in n-\epsilon}{2(1+n)}, \quad \mathrm{y} \quad r^{c}=\frac{n+2-\epsilon\left(1+n^{2}\right)}{2(1+n)}
$$

Los bancos mantienen reservas excesivas, los depósitos son mayores al óptimo y el crédito es menor. Un mercado de dinero donde el banco central interviene mediante operaciones de mercado abierto puede lograr un avance. Ese mercado operaría una vez que la información se asimilara. En principio, parece fácil determinar una regla de política económica que implementara el crédito óptimo. Por ejemplo, ofrecer un crédito máximo por banco igual a $\in$ a la tasa de mercado sería suficiente. En la práctica esto no sería 
eficiente, porque los bancos tienen incentivos para expandir el crédito y el banco central no puede observar el nivel de crédito.

Existe una regla simple de intervención que alcanza el nivel óptimo de crédito a medida que el número de bancos se acerca a infinito, las tasas de interés diarias también convergen al óptimo. Sin embargo, las tasas de interés tendrían mayor varianza. Considérese la siguiente regla para el banco central contingente al balance del sistema ${ }^{1}$ :

1. Cuando el sistema incurre en un sobregiro, ofrece en el mercado "lechero" un monto de crédito igual al sobregiro a la tasa $\left(\bar{r}^{B}\right)$ por encima de la tasa activa de mercado del día anterior: $r^{B}=r^{C}+\delta ; \delta>0$

2. Cuando el sistema tiene un superávit, ofrece una tasa sobre depósitos, sólo para el monto del superávit, a la tasa $\left(\underline{r^{B}}\right)$ por debajo de la tasa pasiva de mercado del día anterior: $r^{B}=r^{d}-\delta$.

El problema para el banco central puede expresarse como:

$$
\begin{aligned}
& \max _{r^{B}, r^{B}} E\left[S W\left(C^{S B}, D^{S B}\right)\right] \\
& \text { s.t. }\left\{C_{i}^{S B}\left(\bar{r}^{B}, \underline{r^{B}}\right), D_{i}^{S B}\left(\bar{r}^{B}, \underline{r}\right)\right\} \in \underset{C_{i} D_{i}}{\arg \max } E\left[\pi_{i}\right]
\end{aligned}
$$

Resolviendo primero la restricción dada la regla del banco central:

$$
\max _{D_{i} C_{i}}(1-\mathrm{C}) \mathrm{C}_{\mathrm{i}}-D D_{i}+p\left(D_{i}-C_{i}-\epsilon_{i}\right) \bar{r}^{m}+(1-p)\left(D_{i}-C_{i}+\epsilon_{i}\right) \underline{r^{m}}
$$

\footnotetext{
${ }^{1}$ En esta regla se asume implícitamente que la determinación de las tasas de interés en el mercado es tal que $r^{B}$ y $\underline{r}^{B}$ son las tasas de equilibrio de la subasta del banco central y son iguales a aquellas obtenidas en el mercado a través de la negociación de los agentes.
} 
Las condiciones de primer orden que resultan son:

$$
\begin{aligned}
& C_{i}=\frac{1-p \bar{r}^{m}+(1-p) \underline{r^{m}}}{n+1} \\
& D_{i}=\frac{p \bar{r}^{m}+(1-p) \underline{r^{m}}}{n+1}
\end{aligned}
$$

Donde $r^{m} y \underline{r^{m}}$ son las tasas esperadas en el mercado "lechero" dado un sobregiro oi un superávit en el i-ésimo banco respectivamente. Para el caso de $n=2$ tenemos que:

$$
\begin{aligned}
& r^{m}=p \bar{r}^{B}+(1-p) \hat{r} \\
& \underline{r}^{m}=(1-p) \underline{r}^{B}+p \hat{r}
\end{aligned}
$$

donde $r$ es la tasa en el mercado "lechero" cuando el sistema terminó balanceado ${ }^{2}$. El resultado óptimo para el banco central es cuando crédito es igual a depósitos. Tomando en cuenta la restricción del problema, obtenemos la siguiente condición:

$$
\bar{r}^{m}+\underline{r^{m}}=
$$

Sustituyendo en (10) se obtiene la condición para la política del banco central:

$$
\bar{r}^{B}+\underline{r^{B}}=2(1-\hat{r})
$$

Un equilibrio simétrico completo, que coincide con el óptimo está dado por:

$$
C_{i}=\frac{1}{2(n+1)}, \quad D_{i}=\frac{1}{2(n+1)}, \quad r^{c}=\frac{n+2}{2(n+1)}, \quad r^{d}=\frac{n}{2(n+1)},
$$

\footnotetext{
${ }^{2}$ Para obtener números sencillos, y sin pérdida de generalidad, podemos suponer que la tasa punitiva es igual a uno. Cuando el sistema está balanceado podemos esperar que, de un equilibrio de Nash, la tasa de interés sea $1 / 2$.
} 


$$
r^{B}=\frac{n+2+\delta}{2(n+1)}, \quad \text { and } \quad \underline{r}^{B}=\frac{n-\delta}{2(n+1)}
$$

Lo cual implica una activa participación del banco central en el mercado "lechero".

\section{CONCLUSIONES}

Este artículo presenta de manera formal un aspecto de la actual discusión en torno a la operación de la política monetaria. Después de dos años de operar de manera innovadora, el Banco de México decidió cambiar esta regla en las operaciones diarias. La razón: la preocupación de que el mercado "lechero" diera incentivos perversos a los bancos comerciales. El argumento desarrollado en este documento es que algunos beneficios de aquella se han desvanecido con la nueva política. Estos beneficios se derivaron de la reducción de la incertidumbre en la demanda de dinero de base enfrentada por el banco central, y pueden separarse en dos partes: La primera se refiere a la liberalización de activos financieros utilizables en proyectos productivos para la reducción de reservas innecesarias en el banco central. La segunda se deriva directamente de la reducción de la incertidumbre a través de la oportunidad de ofrecer la cantidad exacta de dinero que el sistema necesite, y evitando un costo social innecesario. 


\section{BIBLIOGRAFÍA}

Bhattacharya S. \& Douglas Gale, preference Shocks, Liquidity and Central Bank Policy. --?

Cukierman A., 1990. "Why Does the Fed smooth Interest Rates?”, In Michael Belognia (ed.), Monetary Policy on the FED's 75ht Anniversary. Proceedings of the $14^{\text {th }}$. Annual Economic Policy conference of the Federal Reserve Bank of St. Louis. Norwell, MA: Kluwer Academic Publishers.

, 1992. Central Bank Strategy, Credibility and Independence:

Theory and Evidence. The MIT Press.

Friedman, M. \& Anna J. Schwartz, 1963. A Monetary History of the United States, 18671960. Princeton University Press.

Garber P. and Steven R. Weisbrod, "The Economics of Risk in the Payment System" En Haraf and Cagan (ed.) Monetary Policy for a Changing Financial Environment.

Goodhart C., 1988. The Evolution of Central Banks. The MIT Press.

Kashyap, A. \& Jeremy C. Stein, 1993. "Monetary Policy and Bank Lending". National Bureau of Economic Research, Working paper No. 4317.

Summers B., 1994. The payment System: Design, Management and Supervision. 